\title{
Clifford and Harmonic Analysis on Cylinders and Tori
}

\section{Rolf Sören Kraußhar and John Ryan}

\begin{abstract}
Cotangent type functions in $\mathbb{R}^{n}$ are used to construct Cauchy kernels and Green kernels on the conformally flat manifolds $\mathbb{R}^{n} / \mathbb{Z}^{k}$ where $1 \leq k \leq n$. Basic properties of these kernels are discussed including introducing a Cauchy formula, Green's formula, Cauchy transform, Poisson kernel, Szegö kernel and Bergman kernel for certain types of domains. Singular Cauchy integrals are also introduced as are associated Plemelj projection operators. These in turn are used to study Hardy spaces in this context. Also the analogues of CalderónZygmund type operators are introduced in this context, together with singular Clifford holomorphic, or monogenic, kernels defined on sector domains in the context of cylinders. Fundamental differences in the context of the $n$-torus arising from a double singularity for the generalized Cauchy kernel on the torus are also discussed.
\end{abstract}

\section{Introduction}

Classical harmonic analysis has long been applied to the theory of pde's, in particular to solve boundary value problems, for instance the Dirichlet problem. Here we are thinking in particular of solving Laplace's equation over a domain in $\mathbb{R}^{n}$ with suitably smooth boundary and for $L^{p}$ data on the boundary. In recent times it has proved useful to regard some of the operators used to solve such a problem, for instance the double layer potential operator, as the real part of Clifford algebra valued operators. This then links such operators to a Dirac operator, which in turn may be considered as a generalized Cauchy-Riemann operator. Indeed the generalized CauchyRiemann operator used by Stein [24], and Stein and Weiss [25] is a special case of this Dirac operator.

2000 Mathematics Subject Classification: 30G35, 42B30, 53C27, 58J32.

Keywords: Dirac operator, Clifford analysis, cotangent functions. 
The idea to Cliffordize standard operators arising in classical harmonic analysis and elsewhere is due to Coifman and was carried out by a number of authors including McIntosh et al [15]. This provides a fundamental link between classical harmonic analysis and Clifford analysis. In turn a number of basic operators arising in Clifford analysis, including the Dirac operator, the Cauchy transform over hypersurfaces and Plemelj projection operators are all invariant under conformal, or Möbius, transformations, see [22] for details. This invariance is best described using a construction due to Ahlfors [1].

Though not everything arising in Clifford analysis has a conformal invariance it is the case that usually enough remains conformally invariant so that those operators and tools that are not directly invariant under conformal transformations may be reconstructed from operators that are conformally invariant. This suggests that Clifford analysis is intimately linked to the conformal groups and in particular the analogues of Riemann surfaces in Clifford analysis are conformally flat manifolds. These are manifolds which possess an atlas whose chart maps are Möbius transformations, or equivalently Riemannian manifolds whose Weyl tensor vanishes [23]. These supply a relatively rich class of manifolds whose simplest examples include the sphere, hyperbolas, cylinders and the $n$-torus. For the first two cited examples the conformal structure is given via Cayley transformations while in the cases of cylinders and the $n$-torus the conformal structure is given purely by translations.

An overall goal is to develop Clifford analysis over conformally flat manifolds and to link with geometric invariants and properties of these manifolds. For the case of the sphere and hyperbolas this has been partially developed in $[16,27]$ and elsewhere. Here we will deal with the simpler cases of cylinders and tori. It should though be pointed out that Dirac operators and associated Cauchy integral formulas have been introduced in a very general setting in $[5,18,7]$. The approach taken here and in [16] afford a more concrete viewpoint. The general intention is to find as explicit an approach to Clifford analysis as possible for reasonable choices of manifolds. Conformally flat manifolds seems to provide a very good setting to develop this theme and hopefully points the way to proceed in more general settings.

In order to introduce Clifford analysis on cylinders and tori we make use of the fact that the universal covering space of all of these manifolds is $\mathbb{R}^{n}$. So in fact one can easily develop the appropriate analysis on $\mathbb{R}^{n}$ and provided the functions and kernels are $k$-periodic for some $k \in\{1, \ldots, n\}$ then one may project down to the manifold to obtain the equivalent function or kernel in that setting too. So in order, for instance to construct a generalized 
Cauchy kernel in the context of a cylinder or torus one needs to take the Cauchy kernel in $\mathbb{R}^{n}$ and adapt it to construct a new kernel that is $k$-fold periodic. This is done independently in [10], where generalized Eisenstein series are used to construct vector valued cotangent functions. The Cauchy kernel acting on the $L^{p}$ space of a hypersurface embedded in $\mathbb{R}^{n}$ may be regarded as a kernel of Calderón-Zygmund type. In order to extend kernels of Calderón-Zygmund type to the context considered here one adapts the Eisenstein series argument developed in [10]. Results in this direction have been obtained for the particular case $k=n-1$ in [19] where a number of results worked out by McIntosh et al [17, 15, 14] over sector domains are developed essentially in the context of Lipschitz perturbations of the $(n-1)$-torus within a cylinder.

In this paper we begin by introducing a Cauchy integral formula over cylinders and the $n$-torus. This in turn is used to show how a number of basic tools in Clifford analysis including Plemelj projection operators, Szegö and Poisson kernels may be constructed over domains on cylinders. The Poisson kernel is specifically constructed in the context where the domain is a half cylinder. Adapting arguments given in [6] we may also explicitly construct Bergman kernels for harmonic functions defined on a half cylinder.

We conclude by investigating the case of the $n$-torus. The Cauchy kernel here is introduced in [10]. In this case the kernel has two singularities. This gives rise to some fundamental differences from $\mathbb{R}^{n}$ when attempting to construct Hardy spaces in this context. We describe these differences here.

A main key in this paper has been to factor out $\mathbb{R}^{n}$ by a Kleinian group. The Kleinian group in this context is just an integer lattice. However, this raises the issue of taking a more general domain in $\mathbb{R}^{n}$ or $S^{n}$ and factoring this domain by a more general Kleinian group. In this way one constructs other examples of conformally flat manifolds, see for instance [23]. One can now ask if the constructions developed here and in [16] extend to this more general context. This analysis will be developed elsewhere.

\section{Preliminaries}

As in [4] and elsewhere we will consider $\mathbb{R}^{n}$ to be embedded in the real $2^{n}$-dimensional Clifford algebra $C l_{n}$. Here we assume that if $e_{1}, \ldots, e_{n}$ is an orthonormal basis for $\mathbb{R}^{n}$ then under Clifford algebra multiplication $e_{j}^{2}=-1$ for $1 \leq j \leq n$.

Clifford analysis in turn deals with Clifford algebra valued functions that solve Dirac type equations on manifolds. For our needs we first recall the following basic definition. 
Definition 1 ([4]) Suppose that $U$ is a domain in $\mathbb{R}^{n}$ then a $C^{1}$ function $f: U \rightarrow C l_{n}$ is called left monogenic, or left Clifford holomorphic, if for each $x \in U$ we have $\mathbf{D} f(x)=0$ where $\mathbf{D}$ is the Dirac operator $\sum_{j=1}^{n} e_{j} \frac{\partial}{\partial x_{j}}$. A function $g: U \rightarrow C l_{n}$ is called right monogenic, or right Clifford holomorphic, if for each $x \in U$ we have $g(x) \mathbf{D}=0$.

An example of a function that is both left and right monogenic is the generalized Cauchy kernel $G_{1}(x-y)$, where

$$
G_{1}(x)=\frac{x}{\|x\|^{n}}
$$

being the fundamental solution to the Euclidean Dirac operator.

It is a simple but crucial fact that $\mathbf{D}^{2}=-\triangle_{n}$, the Euclidean Laplacian in $\mathbb{R}^{n}$. For $n>2$, the fundamental solution to $\Delta_{n}$ is thus simply

$$
G_{2}(x)=\mathbf{D} G_{1}(x)=\frac{1}{n-2} \frac{1}{\|x\|^{n-2}}
$$

and $G_{2}(x-y)$ is the harmonic Green kernel function.

For $1 \leq k \leq n$ we define the $k$-cylinder $C_{k}$ to be the $n$-dimensional manifold $\mathbb{R}^{n} / \mathbb{Z} e_{1}+\cdots+\mathbb{Z} e_{k}$. When $k=n$ we obtain the $n$-torus $T_{n}$.

It should be noted that for each $k$ the space $\mathbb{R}^{n}$ is the universal covering of $C_{k}$ and consequently there is a projection map $p_{k}: \mathbb{R}^{n} \rightarrow C_{k}$. Though we have chosen a particular lattice $\mathbb{Z} e_{1}+\cdots+\mathbb{Z} e_{k}$ it should be noted that we can in fact work with arbitrary integer lattices lying in $\mathbb{R}^{k}$. Most of the results we present here automatically carry over to this more general context. For convenience we will stick to the integer lattice that we have just constructed.

An open subset $U$ of $\mathbb{R}^{n}$ is said to be $k$-fold periodic if for each $x \in U$ the point $x+m_{1} e_{1}+\cdots+m_{k} e_{k} \in U$ for each $m_{1}, \ldots m_{k} \in \mathbb{Z}$. In this case the set $p_{k}(U)=U^{\prime}$ is an open subset of $C_{k}$. We are interested in the cases where $U^{\prime}$ is a domain. Furthermore suppose that $U$ is a $k$-fold periodic open set then $f: U \rightarrow C l_{n}$ is a $k$-fold periodic function if for each $x \in U$ we have that $f(x)=f\left(x+m_{1} e_{1}+\cdots+m_{k} e_{k}\right)$ for each $m_{1}, \ldots, m_{k} \in \mathbb{Z}$. Examples of such functions include

$$
\cot _{1, k}(x)=\sum_{\left(m_{1}, \ldots m_{k}\right) \in \mathbb{Z}^{k}} G_{1}\left(x+m_{1} e_{1}+\cdots+m_{k} e_{k}\right)
$$

where $1 \leq k \leq n-2$, and

$$
\cot _{2, k}(x)=\sum_{\left(m_{1}, \ldots, m_{k}\right) \in \mathbb{Z}^{k}} G_{2}\left(x+m_{1} e_{1}+\cdots+m_{k} e_{k}\right)
$$

where $1 \leq k \leq n-3$. 
These functions together with some of their basic properties including the locally uniform convergence of the series are introduced in [10] and [11]. They are special cases of generalized higher dimensional Eisenstein series. The functions $\cot _{1, k}$ and $\cot _{2, k}$ are the $k$-fold periodic monogenic (resp. harmonic) generalizations of the classical complex-analytic cotangent function and can be characterized by a generalized cotangent double angle formula in complete analogy to the classical case. See [10] for details. We have the relationship

$$
\mathbf{D} \cot _{2, k}(x)=\cot _{1, k}(x) \text {. }
$$

These functions are defined on the $k$-fold periodic domain $\mathbb{R}^{n} / \mathbb{Z} e_{1}+\cdots+\mathbb{Z} e_{k}$ and take their values in $\mathbb{R}^{n}$ and $\mathbb{R}$ respectively. From now on we shall denote the lattice $\mathbb{Z} e_{1}+\cdots+\mathbb{Z} e_{k}$ by $\mathbb{Z}^{k}$ and a member of this lattice will be denoted by $\mathbf{m}$.

It should be noted that if $f: U \rightarrow C l_{n}$ is a $k$-fold periodic function then the projection $p_{k}$ induces a well defined function $f^{\prime}: U^{\prime} \rightarrow C l_{n}$ defined by $f\left(p_{k}^{-1}\left(x^{\prime}\right)\right)$ for each $x^{\prime} \in U^{\prime}$. Moreover any function $f^{\prime}: U^{\prime} \rightarrow C l_{n}$ lifts to a $k$-fold periodic function $f: U \rightarrow C l_{n}$, where $U=p_{k}^{-1}\left(U^{\prime}\right)$.

For $x, y \in \mathbb{R}^{n} \backslash \mathbb{Z}^{k}$ the functions $\cot _{1, k}(x-y)$ and $\cot _{2, k}(x-y)$ induce functions $\cot _{1, k}^{\prime}\left(x^{\prime}, y^{\prime}\right)$ and $\cot _{2, k}^{\prime}\left(x^{\prime}, y^{\prime}\right)$ where $x^{\prime}=p_{k}(x)$ and $y^{\prime}=p_{k}(y)$. These functions are defined on

$$
\left(C_{k} \times C_{k}\right) \backslash \operatorname{diag}\left(C_{k} \times C_{k}\right)
$$

where

$$
\operatorname{diag}\left(C_{k} \times C_{k}\right)=\left\{\left(x^{\prime}, x^{\prime}\right): x^{\prime} \in C_{k}\right\} .
$$

The projection map $p_{k}$ induces a projection of the Dirac operator $\mathbf{D}$ to a differential operator $D^{\prime}$ acting on differentiable functions defined on domains on $C_{k}$. We shall also call this operator a Dirac operator.

Definition 2 Suppose that $U^{\prime}$ is a domain on $C_{k}$. A function $f^{\prime}: U^{\prime} \rightarrow C l_{n}$ is called cylindrical left monogenic if for each $x^{\prime} \in U^{\prime}$ we have $D^{\prime} f^{\prime}\left(x^{\prime}\right)=0$, while a function $g^{\prime}: U^{\prime} \rightarrow C l_{n}$ is called cylindrical right monogenic if $g^{\prime}\left(x^{\prime}\right) D^{\prime}=0$ for each $x^{\prime} \in U^{\prime}$.

Clearly the function $\cot _{1, k}^{\prime}\left(x^{\prime}, y^{\prime}\right)$ is cylindrical left and right monogenic in both variables $x^{\prime}$ and $y^{\prime}$.

In fact all powers $\mathbf{D}^{l}$ of the Dirac operator $\mathbf{D}$ induce a differential operator $D^{\prime l}$ acting on differentiable functions defined on domains on $C_{k}$. When $l=2$ we get a Laplacian, $\triangle_{n}^{\prime}$, and any solution to the equation $\triangle_{n}^{\prime} f^{\prime}=0$ is called a cylindrical harmonic function. In particular $\cot _{2, k}\left(x^{\prime}, y^{\prime}\right)$ is cylindrical harmonic in both the variables $x^{\prime}$ and $y^{\prime}$. 
Remark: Remember that the absolute convergence abscissa of the function series (2.3) and (2.4) are $k=n-2$, respectively $k=n-3$. One obtains a further degree of convergence by simply coupling certain antipodal lattice points. Following [10], let us divide the lattice $\mathbb{Z}^{k}$ into the following three parts: First there is the origin $\{\mathbf{0}\}$, then let us take the remainder of the lattice and divide that into two equal disjoint parts,

$\Lambda_{k}=\left\{m_{1} e_{1}+\cdots+m_{k} e_{k}: m_{1}, \ldots, m_{k} \in \mathbb{Z}\right.$ and $\left.m_{k}>0\right\} \cup \cdots \cup\left\{m_{1} e_{1}: m_{1}>0\right\}$

and $-\Lambda_{k}$. Note that $\mathbf{0} \notin \Lambda_{k}$. The series

$$
\cot _{1, n-1}(x)=G_{1}(x)+\sum_{\mathbf{m} \in \Lambda_{n-1}}\left(G_{1}(x-\mathbf{m})+G_{1}(x-\mathbf{m})\right)
$$

provides us with the canonical $(n-1)$-fold periodic monogenic generalization of the classical cotangent function in the sense of its characterizability by a generalized cotangent double angle formula, as explained in [10]. The projection map $p_{n-1}$ induces a well-defined cylindrical left monogenic function on $C_{n-1}$.

We further formally define the harmonic $(n-2)$-fold periodic cotangent function $\cot _{2, n-2}(x)$ to be

$$
G_{2}(x)+\sum_{\mathbf{m} \in \Lambda_{n-2}}\left(G_{2}(x-\mathbf{m})+G_{2}(x-\mathbf{m})\right) .
$$

This series in turn defines an $(n-2)$-fold periodic harmonic function in $x$ and via the projection map $p_{n-2}$ we now have a cylindrical harmonic function on the cylinder $C_{n-2}$.

\section{Some integral formulas on cylinders}

Next we want to establish generalizations of some important integral formulas from classical function theory, such as the Cauchy and the Green integral formula, in the context of higher dimensional cylinders and tori. We shall see that the torus $(k=n)$ will play a special role in this context. This is due to the fact that the torus has a special topological structure among all manifolds $C_{k}$; all the other cylinders $C_{k}$ with $k<n$ have an infinite extension in contrast to that of the torus. This difference reflects then consequently in a number of function theoretic aspects, as we shall illustrate explictly in the further development of this paper.

For this reason we proceed to treat the cylinders $C_{k}$ with $k<n$ and the torus $k=n$ in apart sections. We first start with the development of the theory for the cylinders $C_{k}, k<n$. 
By the standard Cauchy Theorem and Cauchy Integral Formula arising in Clifford analysis, see [1] and elsewhere we may readily deduce:

Theorem 1 Suppose that $V$ is a bounded domain in $\mathbb{R}^{n}$ with the property that for each $x \in \mathrm{cl} V$, the closure of $V$, the shifted lattice $x+\mathbb{Z}^{k}$ intersected with $V$ only contains the point $x$. Suppose further that $\partial V$, the boundary of $V$ is Lipschitz continuous and that $f$ is a left monogenic function in an open neighborhood, $U$, of $c l V$. Then for $1 \leq k \leq n-1$ and each $y \in U$

$$
f(y)=\frac{1}{\omega_{n}} \int_{\partial V} \cot _{1, k}(x-y) n(x) f(x) d \sigma(x),
$$

where $\omega_{n}$ is the surface area of the unit sphere in $\mathbb{R}^{n}, n(x)$ is the outer normal vector to $\partial V$ at $x$ and $\sigma$ is the Lebesgue measure of the hypersurface $\partial V$.

The case we are most interested is the one where the open neighborhood $U$ of $V$ is $k$-fold periodic and $f$ is $k$-foldperiodic and monogenic on $U$. In this case we may use the projection map $p_{k}$ to readily obtain the following Cauchy Integral Formula for left cylindrical monogenic functions.

Theorem 2 Suppose $V^{\prime}$ is a subdomain of a domain $U^{\prime}$ lying in $C_{k}$ and $V^{\prime}$ has compact closure. Moreover $c l V^{\prime} \subset U^{\prime}$ and $p_{k}^{-1}\left(\partial V^{\prime}\right)$ is a Lipschitz hypersurface. Suppose also that $f^{\prime}: U^{\prime} \rightarrow C l_{n}$ is a cylindrical left monogenic function and $1 \leq k \leq n-1$ then for each $y \in V^{\prime}$

$$
f^{\prime}\left(y^{\prime}\right)=\frac{1}{\omega_{n}} \int_{\partial V^{\prime}} \cot _{1, k}^{\prime}\left(x^{\prime}, y^{\prime}\right)\left(D_{x} p_{k} n(x)\right) f^{\prime}\left(x^{\prime}\right) d \sigma^{\prime}\left(x^{\prime}\right),
$$

where $x^{\prime}=p_{k}(x), D_{x} p_{k}$ is the derivative of $p_{k}$ at $x$ and $\sigma^{\prime}=p_{k}(\sigma)$.

A similar result also holds for cylindrically right monogenic functions.

Using Cauchy's Theorem and $k$-fold periodicity it may be observed for each $k$-periodic left monogenic function

$$
f: U \rightarrow C l_{n}
$$

defined on a $k$-periodic domain $U$ containing 0 , that

$$
f(0)=\frac{1}{\omega_{n}} \int_{S_{k}(r)} f(x) d \sigma(x)
$$

where $S_{k}(r)$ is the intersection of the $(n-1)$-dimensional sphere of radius $r$ and center 0 with $U$ and the closed strip

$$
S_{k}=\left[-\frac{1}{2}, \frac{1}{2}\right] e_{1} \times \cdots \times\left[-\frac{1}{2}, \frac{1}{2}\right] e_{k} \times \operatorname{span}\left\{e_{k+1}, \ldots, e_{n}\right\} .
$$

The projection $p_{k}$ allows one to construct a similar mean value formula for cylindrically left monogenic functions. 
Suppose now that $\Sigma$ is a sufficiently smooth hypersurface lying in $C_{k}$ and $U^{\prime}$ is a domain whose boundary is $\Sigma$. Suppose also that $\psi$ is a $C l_{n}$ valued function belonging to $L^{p}(\Sigma)$. Then one may readily determine that the integral

$$
\int_{\Sigma} \cot _{1, k}^{\prime}\left(x^{\prime}, y^{\prime}\right)\left(D_{x} p_{k} n(x)\right) \psi\left(x^{\prime}\right) d \sigma^{\prime}\left(x^{\prime}\right)
$$

defines a cylindrical left monogenic function $f^{\prime}\left(y^{\prime}\right)$ on $U$ as $y^{\prime}$ varies over $U^{\prime}$. Note that we are not necessarily assuming $\Sigma$ to be connected. It may also be readily determined that $f^{\prime}$ lifts to a $k$-fold periodic left monogenic function defined on the $k$-fold periodic open set $U=p_{k}^{-1}\left(U^{\prime}\right)$. One is most interested in the cases where $U$ is a domain.

Also the projection map $p_{k}$ readily gives the following version of the Borel-Pompeiu formula.

Theorem 3 Suppose that $V^{\prime}$ is a domain in $C_{k}$ with compact closure and suitably smooth boundary. Suppose also that $\theta: c l V^{\prime} \rightarrow C l_{n}$ is a continuous function and that $\left.\theta\right|_{V^{\prime}}$ is $C^{1}$. Then for each $y^{\prime} \in V^{\prime}$

$$
\begin{gathered}
\theta\left(y^{\prime}\right)=\frac{1}{\omega_{n}}\left(\int_{\partial V^{\prime}} \cot _{1, k}^{\prime}\left(x^{\prime}, y^{\prime}\right)\left(D_{x} p_{k} n(x)\right) \theta(x) d \sigma^{\prime}\left(x^{\prime}\right)\right. \\
\left.+\int_{V^{\prime}} \cot _{1, k}^{\prime}\left(x^{\prime}, y^{\prime}\right) D^{\prime} \theta\left(x^{\prime}\right) d \mu\left(x^{\prime}\right)\right)
\end{gathered}
$$

where $\mu$ is the projection of Lebesgue measure on $\mathbb{R}^{n}$ onto $C_{k}$.

Furthermore if $U^{\prime}$ is a subdomain of $C_{k}$ with compact closure and $\lambda$ : $U^{\prime} \rightarrow C l_{n}$ is an $L^{p}$ function with $1<p<\infty$ then

$$
D^{\prime} \frac{1}{\omega_{n}} \int_{U^{\prime}} \cot _{1, k}^{\prime}\left(x^{\prime}, y^{\prime}\right) \lambda\left(x^{\prime}\right) d \mu\left(x^{\prime}\right)=\lambda\left(y^{\prime}\right)
$$

for each $y^{\prime} \in U^{\prime}$.

One may also use the functions $\cot _{2, k}^{\prime}$ to derive the following version of Green's formula for cylindrically harmonic functions.

Theorem 4 Suppose that $1 \leq k \leq n-2$ and $h: U^{\prime} \rightarrow C l_{n}$ is a cylindrically harmonic function on the domain $U^{\prime} \subset C_{k}$. Suppose also that $V^{\prime}$ is a relatively compact subdomain of $U^{\prime}$ and that $c l V^{\prime} \subset U^{\prime}$. Then provided the boundary of $V^{\prime}$ is sufficiently smooth

$$
\begin{aligned}
h(y)=\frac{1}{\omega_{n}} \int_{\partial V^{\prime}} & \left(\cot _{1, k}^{\prime}\left(x^{\prime}, y^{\prime}\right)\left(D_{x} p_{k} n(x)\right) h(x)\right. \\
& \left.\quad+\cot _{2, k}^{\prime}\left(x^{\prime}, y^{\prime}\right)\left(D_{x} p_{k} n(x)\right) D^{\prime} h\left(x^{\prime}\right)\right) d \sigma^{\prime}\left(x^{\prime}\right)
\end{aligned}
$$

for each $y^{\prime} \in V^{\prime}$. 
Remark: Notice that the series

$$
G_{2}(x-y)+\sum_{\mathbf{m} \in \Lambda_{n-1}} G_{2}(x-y-\mathbf{m})+G_{2}(x-y+\mathbf{m})
$$

is not convergent. A further coupling of antipodal points does not provide a further convergence degree, either.

So it would appear at the very first that one cannot find a Green's formula for cylindrically harmonic functions defined on domains in $C_{n-1}$. However, as we shall see later on, a local one can be obtained for $k=n-1$ if one uses a kernel function with two singularities.

\section{Hardy Spaces and Harmonic Functions on Cylinders}

\subsection{Plemelj projection formulas}

Suppose that $\phi: \operatorname{span}\left\{e_{1}, \ldots, e_{n-1}\right\} \rightarrow R$ is a Lipschitz continuous function. Let $\Sigma$ be its graph

$$
\left\{x_{1} e_{1}+\cdots+x_{n-1} e_{n-1}+\phi\left(x_{1} e_{1}+\cdots+x_{n-1} e_{n-1}\right) e_{n}\right\} .
$$

We say that $\Sigma$ is $k$-fold periodic if $\Sigma=\mathbf{m}+\Sigma$ for each $\mathbf{m} \in \mathbb{Z}^{k}$. The hypersurface $\Sigma$ bounds two domains,

$\Sigma^{+}=\left\{x+x_{n} e_{n}: x \in \Sigma\right.$ and $\left.x_{n}>0\right\}$ and $\Sigma^{-}=\left\{x+x_{n} e_{n}: x \in \Sigma\right.$ and $\left.x_{n}<0\right\}$.

If the hypersurface $\Sigma$ is $k$-fold periodic then so are the domains $\Sigma^{ \pm}$provided $k<n$. For the rest of this section we will assume that $k<n$. If $\Sigma$ is $k$-fold periodic then $\Sigma^{\prime}=p_{k}(\Sigma)$ is a well defined hypersurface in $C_{k}$. We shall call such a hypersurface a Lipschitz surface too. The Lipschitz surface $\Sigma^{\prime}$ bounds two domains $\Sigma^{\prime \pm}=p_{k}\left(\Sigma^{ \pm}\right)$in $C_{k}$.

Suppose now that $\psi^{\prime}: \Sigma^{\prime} \rightarrow C l_{n}$ is $L^{p}$ integrable for some $p \in(1, \infty)$. Let us consider the integral

$$
\frac{1}{\omega_{n}} \int_{\Sigma^{\prime}} \cot _{1, k}^{\prime}\left(x^{\prime}, y^{\prime}\right)\left(D_{x} p_{k}(n(x)) \psi^{\prime}\left(x^{\prime}\right) d \sigma^{\prime}\left(x^{\prime}\right)\right.
$$

where we assume that $y^{\prime} \in \Sigma^{\prime+}$. As we have seen in the previous section this integral defines a cylindrical left monogenic function $\Psi^{\prime+}\left(y^{\prime}\right)$ on $\Sigma^{\prime+}$. Let us now suppose that we have a path $y^{\prime}(t)$ in $\Sigma^{\prime+}$ which tends to infinity as $t$ tends to infinity. We want to know what happens to $\Psi^{\prime+}\left(y^{\prime}(t)\right)$ as $t$ tends to infinity. To determine the answer here and to answer some other questions we will lift back to domains lying in the strip $S_{k}$ in $\mathbb{R}^{n}$. We will denote $\Sigma \cap S_{k}$ by $\Sigma_{k}$ and the relatively open set $\Sigma^{ \pm} \cap S_{k}$ by $\Sigma_{k}^{ \pm}$. We also denote the 
function $\psi^{\prime}\left(p_{k}(x)\right)$ for $x \in \Sigma_{k}$ by $\psi_{k}(x)$. Note that $\psi_{k} \in L^{p}\left(\Sigma_{k}\right)$. We extend this function to the rest of $\Sigma$ by placing $\psi_{k}(x)=0$ for all $x \in \Sigma \backslash \Sigma_{k}$. Thus $\psi_{k} \in L^{p}(\Sigma)$. Under the projection $p_{k}$ the cylindrical left monogenic function $\Psi^{\prime \pm}\left(x^{\prime}\right)$ readily lifts to the left monogenic function $\Psi^{ \pm}(x)$ defined on $\Sigma_{k}^{ \pm}$ respectively. Moreover,

$$
\Psi^{ \pm}(y)=\frac{1}{\omega_{n}} \int_{\Sigma_{k}} \cot _{1, k}(x-y) n(x) \psi_{k}(x) d \sigma(x) .
$$

Let us now suppose that $y(t)$ is a path in $\Sigma_{k}^{+}$that tends to infinity as $t$ tends to infinity. In particular the $n$th co-ordinate $y_{n}(t)$ of $y(t)$ tends to infinity as $t$ tends to infinity. Let us consider $\lim _{t \rightarrow \infty} \Psi^{+}(y(t))$.

Lemma 1 We have

$$
\lim _{t \rightarrow \infty} \Psi^{+}(y(t))=0
$$

Proof. First recall that in the cases $k<n-1$,

$$
\cot _{1, k}(x-y)=\sum_{\mathbf{m} \in \mathbb{Z}^{k}} G_{1}(x-y-\mathbf{m})
$$

and in the case $k=n-1$,

$$
\cot _{1, n-1}(x)=G_{1}(x)+\sum_{\mathbf{m} \in \Lambda_{n-1}}\left(G_{1}(x-\mathbf{m})+G_{1}(x-\mathbf{m})\right) .
$$

In the cases $k<n-1$ we thus precisely deal with the expression

$$
\lim _{t \rightarrow \infty} \Psi^{+}(y(t))=\lim _{t \rightarrow \infty} \frac{1}{\omega_{n}} \int_{\sum_{k}} \sum_{\mathbf{m} \in \mathbb{Z}^{k}} G_{1}(x-y(t)-\mathbf{m}) n(x) \psi_{k}(x) d \sigma(x) .
$$

The right side of this expression becomes in turn

$$
\frac{1}{\omega_{n}} \lim _{t \rightarrow \infty} \sum_{\mathbf{m} \in \mathbb{Z}^{k}} \int_{\Sigma_{k}} G_{1}(x-y(t)-\mathbf{m}) n(x) \psi_{k}(x) d \sigma(x) .
$$

But

$$
\lim _{t \rightarrow \infty} \int_{\Sigma_{k}} G_{1}(x-y(t)-\mathbf{m}) n(x) \psi(x) d \sigma(x)=0
$$

for each $\mathbf{m} \in \mathbb{Z}^{k}$. The result follows from Lebesgue's Convergence Theorem. In the case $k=n-1$ we can apply the same argument, but now involving the series (4.3). 
It follows that $\lim _{t \rightarrow \infty} \Psi^{\prime+}\left(y^{\prime}(t)\right)=0$ where $y^{\prime}(t)=p_{k}(y(t))$. Similarly one can show that if $y(t)$ belongs to $\Sigma_{k}^{-}$and the $n$th component $y_{n}(t)$ tends to infinity as $t$ tends to infinity then $\lim _{t \rightarrow \infty} \Psi^{-}(y(t))=0$ and $\lim _{t \rightarrow \infty} \Psi^{\prime-}(y(t))=0$.

For each $u \in \Sigma$ we can place a cone $C^{+}(u)$ in $\Sigma^{+}$or a cone $C^{-}(u)$ in $\Sigma^{-}$. The maximal aperture of these cones is determined by the Lipschitz constant associated to the graph $\Sigma$. Let us denote the sets $p_{k}^{ \pm}(C(u))$ by $C^{\prime \pm}\left(u^{\prime}\right)$ respectively. Here $u^{\prime}=p_{k}(u)$. Now $u^{\prime} \in \Sigma^{\prime}$ and $C^{\prime \pm}\left(u^{\prime}\right) \subset \Sigma^{\prime \pm}$ respectively. The sets $C^{\prime \pm}\left(u^{\prime}\right)$ are the analogues of cones but now in the context of the manifold $C_{k}$. Let us now consider a path $y^{\prime}(t) \in C^{\prime+}\left(x^{\prime}\right)$ such that $\lim _{t \rightarrow 0} y^{\prime}(t)=u^{\prime}$. Such a path is called a nontangential path to $u^{\prime}$. One may similarly set up nontangential paths in $C^{\prime-}\left(u^{\prime}\right)$ We want to consider $\lim _{t \rightarrow 0} \Psi^{\prime+}\left(y^{\prime}(t)\right)$. By similar arguments to those used in one variable complex analysis and Clifford analysis in the Euclidean setting one may determine that

$$
\begin{aligned}
\lim _{t \rightarrow 0} \Psi^{\prime+}\left(y^{\prime}(t)\right) & =\frac{1}{\omega_{n}} \lim _{t \rightarrow 0} \int_{\Sigma^{\prime}} \cot _{1, k}\left(x^{\prime}, y^{\prime}(t)\right)\left(D_{x} p_{k} n(x)\right) \psi^{\prime}\left(x^{\prime}\right) d \sigma^{\prime}\left(x^{\prime}\right) \\
(4.4) & =\frac{1}{2} \psi^{\prime}\left(x^{\prime}\right)+P . V \cdot \frac{1}{\omega_{n}} \int_{\Sigma^{\prime}} \cot _{1, k}^{\prime}\left(x^{\prime}, u^{\prime}\right)\left(D_{x} p_{k} n(x)\right) \psi^{\prime}\left(x^{\prime}\right) d \sigma^{\prime}\left(x^{\prime}\right),
\end{aligned}
$$

for almost all $u^{\prime} \in \Sigma^{\prime}$. Similarly if $y^{\prime}(t)$ is a path in $C^{\prime-}\left(u^{\prime}\right)$ is a path nontangential to $u^{\prime}$ then we may determine that

$\lim _{t \rightarrow 0} \Psi^{\prime-}\left(y^{\prime}(t)\right)=$

$$
=-\frac{1}{2} \psi^{\prime}\left(u^{\prime}\right)+\frac{1}{\omega_{n}} P . V . \int_{\Sigma^{\prime}} \cot _{1, k}^{\prime}\left(x^{\prime}, u^{\prime}\right)\left(D_{x} p_{k} n(x)\right) \psi^{\prime}\left(x^{\prime}\right) d \sigma^{\prime}(x)
$$

for almost all $u^{\prime} \in \Sigma^{\prime}$. We need to know if these singular integrals are well defined. It is enough to show that the singular integral

$$
P . V . \int_{\Sigma_{k}} \cot _{1, k}(x-y) n(x) \psi(x) d \sigma(x)
$$

is $L^{p}$ bounded.

Proposition 1 The operator

$C_{\Sigma_{k}}: L^{p}\left(\Sigma_{k}\right) \rightarrow L^{p}\left(\Sigma_{k}\right): C_{\Sigma_{k}}(\psi)(y)=\frac{1}{\omega_{n}} P . V . \int_{\Sigma_{k}} \cot _{1, k}(x-y) n(x) \psi(x) d \sigma(x)$

is a well defined $L^{p}$ bounded operator for $1<p<\infty$. 
Proof. The integral

$$
\text { P.V. } \int_{\Sigma_{k}} \sum_{\mathbf{m} \in \mathbb{Z}^{k}} G_{1}(x-y-\mathbf{m}) n(x) \psi(x) d \sigma(x)
$$

is only singular for finitely many terms in the cotangent series

$$
\sum_{\mathbf{m} \in \mathbb{Z}^{k}} G_{1}(x-y-\mathbf{m})
$$

for $k<n-1$, or

$$
G_{1}(x)+\sum_{\mathbf{m} \in \Lambda_{n-1}}\left(G_{1}(x-\mathbf{m})+G_{1}(x-\mathbf{m})\right),
$$

for $k=n-1$, respectively. For these few terms the $L^{p}$ boundedness follows from arguments describing the $L^{p}$ boundedness for the double layer potential operator and singular Cauchy transform for Lipschitz graphs in $\mathbb{R}^{n}$ in [17] and elsewhere. For the remaining terms one simply notes that that part of the operator is a convolution with an $L^{\infty}$ function. The result follows.

By adapting standard arguments from one variable complex analysis and using Lemma 1 we have now shown that the operators

$$
\frac{1}{2} I \pm C_{\Sigma^{\prime}}: L^{p}\left(\Sigma^{\prime}\right) \rightarrow L^{p}\left(\Sigma^{\prime}\right)
$$

are well defined mutually annihilating idempotents, where

$$
C_{\Sigma^{\prime}}(\psi) u^{\prime}=\frac{1}{\omega_{n}} P . V . \int_{\Sigma^{\prime}} \cot _{1, k}^{\prime}\left(x^{\prime}, u^{\prime}\right)\left(D_{x} p_{k} n(x)\right) \psi^{\prime}\left(x^{\prime}\right) d \sigma\left(x^{\prime}\right) .
$$

It follows that for $1<p<\infty$

$$
L^{p}\left(\Sigma^{\prime}\right)=H^{p}\left(\Sigma^{\prime+}\right) \oplus H^{p}\left(\Sigma^{\prime-}\right)
$$

where $H^{p}\left(\Sigma^{\prime \pm}\right)$ is the Hardy $p$-space of cylindrical left monogenic functions defined on $\Sigma^{\prime \pm}$ with continuous nontangential $L^{p}$ extension to $\Sigma^{\prime}$.

\subsection{Bergman, Szegö, Poisson and Kerzman Stein kernels}

Consider now the special case where the Lipschitz graph is the trivial one, the span of $e_{1}, \ldots, e_{n-1}$. In this case

$$
\Sigma_{k}^{+}=\left[-\frac{1}{2}, \frac{1}{2}\right] e_{1} \times \cdots \times\left[-\frac{1}{2}, \frac{1}{2}\right] e_{k} \times \operatorname{span}\left\{e_{k+1}, \ldots, e_{n-1}\right\} \times \mathbb{R}^{+} e_{n} .
$$

We denote this half strip by $S_{k}^{+}$and we denote the half cylinder $p_{k}\left(S_{k}^{+}\right)$ by $C_{k}^{+}$. We claim that while in [24] and elsewhere one sees a development of basic ideas in classical harmonic analysis over the half space $\mathbb{R}^{n,+}$ a suitable analogue for this development in the context of cylinders is the half cylinder $C_{k}^{+}$. 
In particular the Szegö kernel $S_{k}\left(x^{\prime}, y^{\prime}\right)$ for $C_{k}^{+}$is the function

$$
\cot _{1, k}^{\prime}\left(x^{\prime}, y^{\prime}\right) D_{x} p_{k}\left(e_{n}\right),
$$

where $x^{\prime} \in \partial C_{k}^{+}$and $y^{\prime} \in C_{k}^{+}$. Furthermore the Poisson kernel for $C_{k}^{+}$is given by twice the real part of the Szegö kernel. So the Poisson kernel for the half cylinder $C_{k}^{+}$is

$$
P_{k}\left(x^{\prime}, y^{\prime}\right)=2 R e\left(\cot _{1, k}\left(x^{\prime}, y^{\prime}\right) D_{x}\left(p_{k} e_{n}\right)\right) .
$$

In fact this follows directly from the Plemelj formula given by equation 1 . This Poisson kernel solves the Dirichlet problem for cylindrically harmonic functions on $C_{k}^{+}$with $L^{p}$ data given on the boundary for $1<p<\infty$.

In the particular case $k=n-1$ we have $\partial C_{n-1}^{+}=T_{n-1}$. The Poisson kernel solves thus the Dirichlet problem on the $n-1$ torus in this context.

By similar arguments to those given in [6] it may be readily shown that the Bergman kernel $B_{k}\left(x^{\prime}, y^{\prime}\right)$ for $L^{2}$ integrable cylindrically monogenic functions defined on the half cylinder $C_{k}^{+}$is given by

$$
2 \frac{\partial}{\partial x_{n}} S_{k}\left(x^{\prime}, y^{\prime}\right)
$$

This function may be readily lifted to obtain the Bergman kernel for $k$-fold periodic square integrable monogenic functions defined on the half strip $S_{k}^{+}$. A Bergman kernel for square integrable left monogenic functions defined on a half strip has previously been introduced in [8]. By similar arguments to those given in [6], which extend the classical quadratic relation between the Bergman and the Szegö kernel (see e.g. [2]) for the particular case of dealing with the half-space, one may deduce that the Bergman kernel for square integrable cylindrical harmonic functions defined on the half cylinder is given by the real part of the Bergman kernel $B_{k}\left(x^{\prime}, y^{\prime}\right)$.

It should be pointed out here that in our construction of the Poisson kernel and Bergman kernel we are explicitly using the integer lattice $\mathbb{Z}^{k}$ as we need $D_{x}\left(p_{k} e_{n}\right)$ to be orthogonal to the boundary of $C_{k}^{+}$.

It should also be mentioned that these explicit formulas for the Bergman kernel remain valid if we consider instead of the $L^{2}$-space the $L^{p}$ space with $2 \leq p<\infty$.

Furthermore, we observe that one can also extend the Kerzman-Stein theory to the setting of cylinders and tori considered here. What follows can be regarded as complementary results to [26] in which a Kerzman-Stein theory has been developed for manifolds on the unit sphere. In [2] for instance the classical Kerzman-Stein theory in one complex variable is well illustrated. For similar results in the framework of monogenic functions in higher dimensional Euclidean space $\mathbb{R}^{n}$ within the context of Lipschitz continuous functions, we refer for example to [3]. 
In what follows let us denote the Cauchy-transform by

$$
\mathcal{H}^{\prime}:=\frac{1}{2} I+C_{\Sigma^{\prime}}
$$

Adapting the calculations from [3] to the context considered here, we finally obtain the following explicit representation formula for the $L^{p}\left(\Sigma^{\prime}\right)$ adjoint of the Cauchy-transform:

$$
\mathcal{H}^{\prime *} \Psi^{\prime}\left(u^{\prime}\right)=\frac{1}{2} \Psi^{\prime}\left(u^{\prime}\right)+\frac{1}{\omega_{n}} P . V . \int_{\Sigma^{\prime}} \overline{\left(D_{u} p_{k} n(u)\right)} \cot _{1, k}^{\prime}\left(x^{\prime}, u^{\prime}\right) \Psi^{\prime}\left(x^{\prime}\right) d \sigma\left(x^{\prime}\right)
$$

which is again $L^{p}\left(\Sigma^{\prime}\right)$ bounded, where here we use the usual Clifford algebra conjugation —as defined in [22] and elsewhere. Following for example the above cited works it is natural to introduce the cylindrical Kerzman-Stein operator as follows:

$$
\mathcal{A}^{\prime} \Psi^{\prime}\left(u^{\prime}\right):=\left[\mathcal{H}^{\prime}-\mathcal{H}^{\prime *}\right] \Psi^{\prime}\left(u^{\prime}\right)=\frac{1}{\omega_{n}} P . V . \int_{\Sigma^{\prime}} A\left(x^{\prime}, u^{\prime}\right) \Psi^{\prime}\left(x^{\prime}\right) d \sigma\left(x^{\prime}\right)
$$

where we put

$$
A\left(x^{\prime}, u^{\prime}\right)=\cot _{1, k}^{\prime}\left(x^{\prime}, u^{\prime}\right)\left(D_{x} p_{k} n(x)\right)-\overline{\left(D_{u} p_{k} n(u)\right)} \cot _{1, k}^{\prime}\left(x^{\prime}, u^{\prime}\right) .
$$

As $\Sigma^{\prime}$ has a Lipschitz boundary, we can directly adapt the argument from [3, Lemma 4.5]. to the context of the cylinder $C_{k}$, and thus, we infer that the operator $I+\mathcal{A}^{\prime}$ is invertible on $L^{p}\left(\Sigma^{\prime}\right)$. As a consequence we can express the Szegö projection $\mathcal{P}^{\prime}$ on the space $L^{p}\left(\Sigma^{\prime}\right)$ in the following way

$$
\mathcal{P}^{\prime}=\mathcal{H}^{\prime}\left(I+\mathcal{A}^{\prime}\right)
$$

In many cases, as for instance in cases when $\Sigma^{\prime}$ is topologically similar to $p_{k}(\partial B), B$ denoting a ball in $\mathbb{R}^{n}$, this formula provides simpler numerical methods to determine the Szegö kernel of the respective domains lying on the surface of the cylinder $C_{k}$ than to determine it directly, for example by a Gram-Schmidt algorithm which is in general numerically very unstable. However, we should mention that in the context of Lipschitz graphs the operator $\mathcal{A}^{\prime}$ is in general no longer a compact operator. It is always a compact operator when the input functions $\Psi$ are $\mu$-Hölder continuous.

One can say more. We start with the following definition.

Definition 3 A hypersurface $\Theta$ in $\mathbb{R}^{n}$ is called strongly Lipschitz if $\Theta$ can be covered by open sets, each of which is a the graph of some Lipschitz function, and each such Lipschitz graph has the same Lipschitz constant. 
We are interested in the case where $\Theta$ is $k$-fold periodic. In this case we consider the projected hypersurface $\Theta^{\prime}=p_{k}(\Theta)$. Then we may determine:

Theorem 5 Suppose that $1 \leq p \leq n-1$ and $\Theta^{\prime}$ is the projection of a $k$-fold periodic strongly Lipschitz graph and $\Theta^{\prime}$ is the boundary of two domains $\Theta^{\prime+}$ and $\Theta^{\prime-}$ within $C_{k}$. Suppose also that $\Theta^{\prime}$ is compact. Then for each $p \in(1, \infty)$

$$
L^{p}\left(\Theta^{\prime}\right)=H^{p}\left(\Theta^{\prime+}\right) \oplus H^{p}\left(\Theta^{\prime-}\right),
$$

where $H^{p}\left(\Theta^{\prime \pm}\right)$ is the Hardy p-space of cylindrical left monogenic functions on $\Theta^{\prime \pm}$ with nontangential $L^{p}$ extension to the boundary $\Theta^{\prime}$.

This result may be obtained by partition of unity arguments and by adapting standard Calderón-Zygmund techniques.

\subsection{Calderón Zygmund type operators}

Let us proceed now to introduce a smooth function

$$
\chi: \operatorname{span}\left\{e_{1}, \ldots, e_{n-1}\right\} \backslash\{0\} \rightarrow C l_{n} .
$$

We shall assume that this function is an odd function and that it is homogeneous of degree zero. Let $l(x)=\frac{\chi(x)}{\|x\|^{n-1}}$ and suppose that

$$
\|l(x-y)-l(x-z)\| \leq C(n) \frac{\|y-z\|}{\|x-z\|^{n}} \text { for } 2\|y-z\|<\|x-z\|
$$

and for some dimensional constant $C(n)$. Then, see [24], the convolution operator

$$
\begin{gathered}
T_{l}: L^{p}\left(\operatorname{span}\left\{e_{1}, \ldots, e_{n-1}\right\}\right) \rightarrow L^{p}\left(\operatorname{span}\left\{e_{1}, \ldots, e_{n-1}\right\}\right): \\
T_{l}(\psi)=\int_{\operatorname{span}\left\{e_{1}, \ldots, e_{n-1}\right\}} l(x-y) \psi(x) d x^{n-1}
\end{gathered}
$$

is an operator of Calderón-Zygmund type, and it is well defined and bounded for $1<p<\infty$. For $1 \leq k \leq n-2$ let us introduce the function

$$
L(x-y)=\sum_{\mathbf{m} \in \mathbb{Z}^{k}} l(x-y-\mathbf{m})
$$

where $x$ and $y$ belong to $\operatorname{span}\left\{e_{1}, \ldots, e_{n-1}\right\}$ and $x \neq y$. Via the projection $p_{k}$ the function $L(x-y)$ projects to give a $C l_{n}$ valued function $L^{\prime}\left(x^{\prime}, y^{\prime}\right)$ defined on $\left(\partial C_{k}^{+} \times \partial C_{k}^{+}\right) \backslash$ diag $\partial C_{k}^{+}$. By very similar arguments to those used to prove Proposition 1 one may determine: 
Theorem 6 For $1<p<\infty$ and $1 \leq k \leq n-2$ the operator

$$
T_{L^{\prime}}^{\prime}: L^{p}\left(\partial C_{k}^{+}\right) \rightarrow L^{p}\left(\partial C_{k}^{+}\right): T_{L^{\prime}}^{\prime}\left(\psi^{\prime}\right)=P . V . \int_{\partial C_{k}^{+}} L^{\prime}\left(x^{\prime}, y^{\prime}\right) \psi^{\prime}\left(x^{\prime}\right) d \sigma^{\prime}\left(x^{\prime}\right)
$$

is a bounded operator.

So in the setting of the cylinders the operator $T_{L^{\prime}}^{\prime}$ is the appropriate analogue of an operator of Calderón-Zygmund type.

Let us now introduce operators of Calderón-Zygmund type acting on the $L^{p}$ space of the $(n-1)$-torus $T_{n-1}$. Let us reconsider the function $l: \mathbb{R}^{n} \backslash\{0\} \rightarrow C l_{n}$ that we introduced earlier. From this function we may introduce the function

$$
L(x, y)=l(x-y)+\sum_{\mathbf{m} \in \Lambda_{n-1}}(l(x-y-\mathbf{m})+l(x-y+\mathbf{m})) .
$$

As

$$
\|l(x-y)-l(x-z)\|<C(n) \frac{\|y-z\|}{\|x-z\|^{n}}
$$

for $2\|y-z\|<\|x-z\|$ this series is locally uniformaly convergent and so the function $L$ is well defined. The projection map $p_{n-1}$ now induces from $L$ a map $L^{\prime}\left(x^{\prime}, y^{\prime}\right)$ and by similar arguments to those used to establish Theorem 6 we now have:

Theorem 7 For $1<p<\infty$ the operator

$$
T_{L^{\prime}}^{\prime}: L^{p}\left(T_{n-1}\right) \rightarrow L^{p}\left(T_{n-1}\right): T_{L^{\prime}}^{\prime}\left(\psi^{\prime}\right) u^{\prime}=P . V . \int_{T_{n-1}} L^{\prime}\left(x^{\prime}, y^{\prime}\right) \psi^{\prime}\left(x^{\prime}\right) d \sigma\left(x^{\prime}\right)
$$

is $L^{p}$ bounded.

The links between Clifford analysis and harmonic analysis associated to $(n-1)$-fold periodic Lipschitz surfaces is described in some detail in [19]. Here we will briefly outline some of this analysis and generalize to the $C_{k}$ setting.

A sector domain $S_{\alpha}$ is defined to be the domain

$\left\{x \in \mathbb{R}^{n}:\left|x_{n}\right|<\alpha\left\|x_{1} e_{1}+\cdots+x_{n-1} e_{n-1}\right\|\right.$ for some fixed $\left.\alpha \in \mathbb{R}^{+}\right\}$.

For $1 \leq k \leq n-1$ the projection $p_{k}\left(S_{\alpha} \cap S_{k}\right)$ is a domain in $C_{k}$. We call this type of domain a cylindrical sector domain and we denote it by $S_{\alpha}^{\prime}$. The complement of $S_{\alpha}$ consists of the union of two closed cones $C_{\alpha}^{ \pm}$with $e_{n} \in C_{\alpha}^{+}$ and $-e_{n} \in C_{\alpha}^{-}$. We denote the domains $S_{\alpha} \cup\left(C_{\alpha}^{ \pm} \backslash\{0\}\right)$ by $S_{\alpha, \pm}$ respectively. We denote the domains $p_{k}\left(S_{\alpha, \pm} \cap S_{k}\right)$ by $S_{\alpha, \pm}^{\prime}$ respectively. Furthermore we denote $p_{k}\left(C_{\alpha}^{ \pm} \cap S_{k}\right)$ by $C_{\alpha}^{\prime \pm}$. 
Following $[15,14,17]$ we shall consider a left and right monogenic function $T(x)$ defined on $S_{\alpha}$. Moreover

$$
\|T(x)\|<C\|x\|^{-(n-1)}
$$

for some constant $C \in \mathbb{R}^{+}$. The monogenic function $T$ is chosen so that there is a bounded measurable function $t: C_{\alpha}^{+} \rightarrow C l_{n}$. In fact $t(u)-t(v)$ is defined to be

$$
\int_{A(u, v)} T(x) n(x) d \sigma(x)
$$

where $A(u, v)$ is a smooth orientable manifold in $S_{\alpha}$ which joins the $(n-2)$ dimensional spheres $S_{u}$ and $S_{v}$, where

$$
S_{u}=\left\{x \in \mathbb{R}^{n}:<x, u>=0,\|x\|=\|u\|\right\}
$$

and $S_{v}$ is defined similarly. Via the projection $p_{k}\left(C_{\alpha}^{+} \cap S_{k}\right)$ the map $t$ induces a bounded measurable function $t^{\prime}: C_{\alpha}^{\prime+} \rightarrow C l_{n}$.

In $[15,14,17]$ it is shown that there are left and right monogenic functions $T^{ \pm}: S_{\alpha}^{ \pm} \rightarrow C l_{n}$ such that

$$
\left\|T^{ \pm}(x)\right\|<C^{ \pm}\|x\|^{1-n} \quad \text { on } S_{\alpha}^{ \pm}
$$

and for some constants $C^{ \pm} \in \mathbb{R}^{+}$. Moreover $T(x)=T^{+}(x)+T^{-}(x)$ on $S_{\alpha}$. Furthermore there are associated to $T^{ \pm}$bounded measurable functions $t_{1}$ and $t_{2}$ defined on $C_{\alpha}^{+}$such that $t_{1}(x)+t_{2}(x)=t(x)$. Again these functions give rise via the projection $p_{k}$ to bounded measurable functions $t_{1}^{\prime}$ and $t_{2}^{\prime}$ defined on $C_{\alpha}^{\prime+}$ and such that $t_{1}^{\prime}\left(x^{\prime}\right)+t_{2}^{\prime}\left(x^{\prime}\right)=t^{\prime}\left(x^{\prime}\right)$. According to Eisenstein [9] (see also [19]) there are at most $2 k m^{k-1}$ entries $\mathbf{m}=m_{1} e_{1}+\cdots+m_{k} e_{k} \in \mathbb{Z}^{k}$ such that $\left|m_{1}\right|+\cdots+\left|m_{k}\right|=m$. Consequently the series

$$
\sum_{\mathbf{m} \in \mathbb{Z}^{k}} T(x-\mathbf{m}) \quad \text { and } \quad \sum_{\mathbf{m} \in \mathbb{Z}} T^{ \pm}(x-\mathbf{m})
$$

are locally uniformly convergent for $1 \leq k \leq n-2$. The particular case $k=n-1$ is treated in [19]. We denote these $k$-fold periodic left and right monogenic functions by $W(x)$ and $W^{ \pm}(x)$ respectively. The projection $p_{k}: S_{k} \rightarrow C_{k}$ gives rise to cylindrical left and right monogenic functions $W^{\prime}\left(x^{\prime}\right)$ and $W^{\prime \pm}\left(x^{\prime}\right)$ defined on $S_{\alpha}^{\prime}$ and $S_{\alpha, \pm}^{\prime}$ respectively. Moreover $W^{\prime+}\left(x^{\prime}\right)+$ $W^{\prime-}\left(x^{\prime}\right)=W^{\prime}\left(x^{\prime}\right)$ on $S_{\alpha}^{\prime}$.

From $W(x)$ and $W^{ \pm}(x)$ we obtain the kernels $W(x-y)$ and $W^{ \pm}(x-y)$. Via $p_{k}$ these functions kernels give rise to kernels $W^{\prime}\left(x^{\prime}, y^{\prime}\right)$ and $W^{\prime \pm}\left(x^{\prime}, y^{\prime}\right)$. Suppose that $\Sigma_{k}^{\prime}$ is the image of a $k$-fold periodic Lipschitz graph $\Sigma$ under the projection $p_{k}$. Suppose also that $\Sigma_{k}^{\prime} \subset S_{\alpha}^{\prime}$. Furthermore for each $x \in \Sigma$ let $B_{\Sigma}(x, r)=\{y \in \Sigma:\|y\|<r\}$. Denote $p_{k}\left(B_{\Sigma}(x, r)\right)$ by $B_{\Sigma^{\prime}}\left(x^{\prime}, r\right)$. By simple adaptations of arguments used in [19] one may now determine: 
Theorem 8 For $1<p<\infty$ The operator

$$
\begin{gathered}
T_{W^{\prime}, t^{\prime}}: L^{p}\left(\Sigma^{\prime}\right) \rightarrow L^{p}(\Sigma): \\
T_{W^{\prime}, t^{\prime}}\left(\psi^{\prime}\right)=\lim _{\varepsilon \rightarrow 0}\left(\int_{\Sigma^{\prime} \backslash B_{\Sigma^{\prime}}\left(x^{\prime}, \epsilon\right)} W^{\prime}\left(x^{\prime}, y^{\prime}\right)\left(D_{x} p_{k} n(x)\right) \psi^{\prime}\left(x^{\prime}\right) d \sigma^{\prime}\left(x^{\prime}\right)\right. \\
+t^{\prime}\left(\epsilon\left(D_{x} p_{k} n(x)\right) \psi^{\prime}\left(y^{\prime}\right)\right)
\end{gathered}
$$

is a bounded operator.

Moreover for $1<p<\infty$ the operators

$$
\begin{gathered}
T_{W^{\prime \pm}, t^{\prime \pm}}: H^{p}\left(\Sigma^{ \pm}\right) \rightarrow H^{p}\left(\Sigma^{ \pm}\right): \\
T_{W^{\prime \pm}, t^{ \pm}}\left(\Psi^{\prime \pm}\right)=\lim _{\epsilon \rightarrow 0}\left(\int_{\Sigma^{\prime} \backslash B_{\Sigma^{\prime}}\left(y^{\prime}, \epsilon\right)} W^{ \pm}\left(x^{\prime}, y^{\prime}\right)\left(D_{x} p_{k} n(x)\right) \Psi^{\prime}\left(x^{\prime}\right) d \sigma\left(x^{\prime}\right)\right. \\
+t^{\prime \pm}\left(\epsilon\left(D_{x} p_{k} n(x)\right) \Psi^{\prime \pm}\left(y^{\prime}\right)\right)
\end{gathered}
$$

are bounded.

\section{Polymonogenic cylindrical functions}

Let us now turn to look at integral representations to the higher order DiracLaplace equation $D^{\prime p} f^{\prime}=0$. The Cauchy-Green kernels for the equation $\mathbf{D}^{p} f=0$ appear in [21]. For $p=1, \ldots, n-1$ they are

$$
G_{2 l+1}(x)=C(p, n) \frac{x}{\|x\|^{n-2 l}}
$$

when $2 l+1=p$ and

$$
G_{2 l}(x)=C(p, n) \frac{1}{\|x\|^{n-2 l}}
$$

when $2 l=p$. The real constants $C(p, n)$ are chosen so that $\mathbf{D} G_{p}=G_{p-1}$. Precise formulas for $G_{p}$ for $p \geq n$ are given in [21]. However, as we shall soon see they do not directly give rise to integral representations for solutions to $D^{\prime p} f^{\prime}=0$.

One formally defines $\cot _{p, k}(x-y)$ to be given by the Mittag-Leffler type series

$$
\sum_{\mathbf{m} \in \mathbb{Z}^{k}} G_{p}(x-y-\mathbf{m})
$$


First it should be pointed out that this series does not converge for $p \geq n$ and this is the reason why we did not bother to specify what the kernels $G_{p}$ are in these cases. Further these series do not converge for all choices of $p$ and $k \in\{1, \ldots, n-1\}$. Let us recall that there are at most $2 \mathrm{~km}^{k-1}$ co-ordinates $\mathbf{m}=m_{1} e_{1}+\cdots+m_{k} e_{k} \in \mathbb{Z}^{k}$ such that $\left|m_{1}\right|+\cdots+\left|m_{k}\right|=m$. It follows that the generalized Eisenstein series for the kernel $\cot _{p, k}(x-y)$ converges locally uniformly for $p \leq n-k-1$. Moreover $\mathbf{D} \cot _{p, k}(x-y)=\cot _{p-1, k}(x-y)$. For the special case $p=n-k$ if we re-define $\cot _{n-k, k}(x-y)$ to by the series

$$
G_{n-k}(x-y)+\sum_{\mathbf{m} \in \Lambda_{k}}\left(G_{n-k}(x-y-\mathbf{m})+G_{n-k}(x-y-\mathbf{m})\right),
$$

as this series is locally uniformly convergent the function $\cot _{n-k, k}(x-y)$ is well defined. Moreover, $\mathbf{D} \cot _{n-k, k}(x-y)=\cot _{n-k-1, k}(x-y)$. See [11] for more details.

In the special case where $n$ is even and $k=1$ the kernel is a solution of the Fueter-Sce equation $\mathbf{D} \triangle_{n^{2}}^{\frac{n-2}{2}} f=0$. When $n$ is odd the series (see also [12])

$$
(x-y)^{-1}+\sum_{\mathbf{m} \in \mathbb{Z}^{+}}\left((x-y-\mathbf{m})^{-1}+(x-y+\mathbf{m})^{-1}\right)
$$

is still locally uniformly convergent and still satisfies the Fueter-Sce equation $\mathbf{D} \triangle_{n}^{\frac{n-2}{2}} f=0$. This time though the operator $\mathbf{D} \triangle_{n}^{\frac{n-2}{2}}$ is a pseudo-differential operator acting on our series in a distributional sense. See [20] for details. It should be noted that for all choices of $n$ the series (5.3) is a solution to the hyperbolic Dirac equation

$$
x_{1} \mathbf{D} f(x)+n P_{e_{1}}(f)(x)=0
$$

where here $f(x)=f_{1} e_{1}+\cdots+f_{n} e_{n}$ and $P_{e_{1}}(f)=f_{1}$. Here we are assuming that $x_{1} \neq 0$. A similar equation to this is described in [13] and elsewhere.

Returning to the functions $\cot _{p, k}(x-y)$ for $p \leq n-k$, we can use the projection map $p_{k}$ to induce functions $\cot _{p, k}^{\prime}\left(x^{\prime}, y^{\prime}\right)$. These functions are annihilated by the operator $D^{\prime p}$ acting on either $x$ or $y$ and either on the left or on the right. It is straightforward to obtain the following result.

Theorem 9 Suppose that $U^{\prime}$ is a domain in $C_{k}$ and $V^{\prime}$ is a relatively compact domain lying in $U^{\prime}$ and $\partial V^{\prime}$ is strongly Lipschitz. Suppose also that $f^{\prime}: U^{\prime} \rightarrow C l_{n}$ satisfies the equation $D^{\prime p} f^{\prime}=0$ for some $p \in\{1, \ldots, n-k\}$ then for each $y^{\prime} \in V^{\prime}$

$$
f\left(y^{\prime}\right)=\frac{1}{\omega_{n}} \int_{\partial V^{\prime}} \sum_{j=1}^{p} \cot _{j, k}^{\prime}\left(x^{\prime}, y^{\prime}\right)\left(D_{x} p_{k} n(x)\right) D^{\prime j-1} f^{\prime}\left(x^{\prime}\right) d \sigma\left(x^{\prime}\right) .
$$




\section{Clifford analysis on the torus}

Last of all we treat the case $k=n$. In this case $C_{k}$ is the $n$-torus $T_{n}$. To do Clifford analysis on the torus, we need to consider $n$-fold periodic functions. In contrast to all the other cases treated earlier in this paper, there is no nontrivial $n$-fold periodic function with the property of being monogenic and of having only one pole of the order of the Euclidean Cauchy kernel in each period cell which is just a bounded parallelepiped in this case. This is a consequence of the Liouville theorem. The sum of the residues of any monogenic $n$-fold periodic function within a period cell vanishes. See [10] for more details. However, it is possible to construct $n$-fold periodic monogenic functions which have two poles of the order of the Euclidean Cauchy kernel per period cell and no further poles.

As a examples serve the following family of functions

$$
\cot _{1, n ; a, b}(x-y)=G_{1}(x-y-a)+G_{1}(x-y-b)+
$$

$+\sum_{\mathbf{m} \in \mathbb{Z}^{n} \backslash\{0\}}\left(G_{1}(x-y-a+\mathbf{m})-G_{1}(x-y-b+\mathbf{m})-G_{1}(\mathbf{m}-a)+G_{1}(\mathbf{m}-b)\right)$

where $a, b \in \mathbb{R}^{n} \backslash \mathbb{Z}^{n}$ and $a \neq b \bmod \mathbb{Z}^{n}$. This family of functions was introduced in [10] and serve as analogues of the cotangent type functions $\cot _{1, k}$ in the context on the $n$-torus.

This series is locally uniformly convergent and defines an $n$-fold periodic left and right monogenic function in both $x$ and $y$. This function has singularities at both $x-y=a$ and $x-y=b$. Using the projection map $p_{n}$ this generalized cotangent function defines a vector valued function $\cot _{1, n, a, b}^{\prime}\left(x^{\prime}, y^{\prime}\right)$.

Definition 4 For a domain $U^{\prime} \subset T_{n}$ a smooth function $f^{\prime}: U^{\prime} \rightarrow C l_{n}$ is said to be toroidal left monogenic if $D^{\prime} f^{\prime}=0$.

A similar definition can be set up for toroidal right monogenic functions. The function $\cot _{1, n, a, b}^{\prime}\left(x^{\prime}, y^{\prime}\right)$ is both toroidal left and right monogenic in both the variables $x^{\prime}$ and $y^{\prime}$.

Suppose $U$ is an $n$-fold periodic open subset of $\mathbb{R}^{n}$ and $f: U \rightarrow C l_{n}$ is $n$-fold periodic. From $f$ we can construct the $n$-fold periodic function

$$
F: U-a \rightarrow C l_{n}: F(x)=f(x+a) .
$$

Via the projection $p_{n}$ we obtain from $f$ a function $F^{\prime}$ defined on an open set $U_{a}^{\prime}=p_{n}(U-a)$. 
From this construction we get the following Cauchy Integral Formula.

Theorem 10 Suppose that $U^{\prime}$ is a domain in $T_{n}$ and that $f^{\prime}: U^{\prime} \rightarrow C l_{n}$ is a toroidal left monogenic function. Suppose also that $V^{\prime}$ is a subdomain of $U^{\prime}$ whose closure also lies in $U^{\prime}$ and $V^{\prime}$ has a strongly Lipschitz boundary. Furthermore $b$ does not belong to the closure of $V_{a}^{\prime}$. Then for each $y^{\prime} \in V^{\prime}$

$$
f^{\prime}\left(y^{\prime}\right)=\frac{1}{\omega_{n}} \int_{\partial V_{a}^{\prime}} \cot _{1, n, a, b}^{\prime}\left(x^{\prime}, y_{a}^{\prime}\right)\left(D_{x} p_{n} n(x)\right) F^{\prime}\left(x^{\prime}\right) d \sigma^{\prime}\left(x^{\prime}\right)
$$

where $y_{a}^{\prime}=p_{n}(y-a)$.

Remark: This Cauchy integral formula has a local character, due to the fact that we are dealing with a Cauchy kernel function that has two point singularities.

Suppose now that $\Sigma$ is an $n$-fold periodic Lipschitz hypersurface in $\mathbb{R}^{n}$. Let $\Sigma^{\prime}$ denote the hypersurface $p_{n}\left(\Sigma^{\prime}\right) \subset T_{n}$, while $\Sigma_{a}^{\prime}$ denotes $p_{n}(\Sigma-a)$ and $\Sigma_{b}^{\prime}=p_{n}(\Sigma-b)$.

Suppose now that $\psi^{\prime} \in L^{p}\left(\Sigma^{\prime}\right)$ for some $p \in(1, \infty)$. Then $\psi_{a}^{\prime}(x),=$ $\psi^{\prime}\left(x_{a}^{\prime}\right)$, belongs to $L^{p}\left(\Sigma_{a}^{\prime}\right)$ where $x_{a}^{\prime}=p_{n}(x-a)$ and $\psi_{b}^{\prime}(x),=\psi^{\prime}\left(x_{b}^{\prime}\right)$ belongs to $L^{p}\left(\Sigma_{b}^{\prime}\right)$ where $x_{b}^{\prime}=p_{n}(x-b)$. We shall assume that $a$ and $b$ do not belong to $\Sigma$. The integral

$$
\frac{1}{\omega_{n}} \int_{\Sigma^{\prime}} \cot _{1, n, a, b}^{\prime}\left(x^{\prime}, y^{\prime}\right)\left(D_{x} p_{n} n(x)\right) \psi^{\prime}\left(x^{\prime}\right) d \sigma^{\prime}\left(x^{\prime}\right)
$$

defines a toroidal left monogenic function on $T_{n} \backslash\left(\Sigma_{a}^{\prime} \cup \Sigma_{b}^{\prime}\right)$. Furthermore if $y^{\prime}(t)$ is a path in $T_{n} \backslash\left(\Sigma_{a}^{\prime} \cup \Sigma_{b}^{\prime}\right)$ with nontangential limit $u^{\prime} \in \Sigma_{a}^{\prime}$ then

$$
\begin{aligned}
\lim _{t \rightarrow 0} & \frac{1}{\omega_{n}} \int_{\Sigma^{\prime}} \cot _{1, n, a, b}^{\prime}\left(x^{\prime}, y^{\prime}(t)\right)\left(D_{x} p_{n}(n(x)) \psi^{\prime}\left(x^{\prime}\right) d \sigma^{\prime}\left(x^{\prime}\right)\right. \\
= & \pm \frac{1}{2} \psi_{a}^{\prime}\left(u^{\prime}\right)+\frac{1}{\omega_{n}} P . V . \int_{\Sigma^{\prime}} \cot _{1, n, a, b}^{\prime}\left(x^{\prime}, u^{\prime}\right)\left(D_{x} p_{n} n(x)\right) \psi^{\prime}\left(x^{\prime}\right) d \sigma^{\prime}\left(x^{\prime}\right)
\end{aligned}
$$

for almost all $u^{\prime} \in \Sigma_{a}^{\prime}$.

A similar formula holds if the path $y^{\prime}(t)$ were to nontangentially approach a point $v^{\prime} \in \Sigma_{b}^{\prime}$. The plus or minus sign appearing in the previous formula depends on the choice of orientation one gives to $\Sigma$ and on which side of $\Sigma_{a}^{\prime}$ the path $y^{\prime}(t)$ approaches $u^{\prime}$. One may readily adapt earlier arguments to show that the singular integral appearing in the previous formula is indeed $L^{p}$ bounded. Also Kerzman-Stein formulas can be obtained for the setting of the $n$-torus $T_{n}$. 
Remark: Notice that we can adapt the construction given by Equation (6.1) to get a local Green's formula for harmonic functions on the $(n-1)$ cylin$\operatorname{der} C_{n-1}$. Namely we replace $G_{1}$ by $G_{2}$ in (6.1). In the same way, namely by replacing $G_{1}$ by $G_{k}$ in (6.1) we obtain for the function class $\mathbf{D}^{k} f=0$ a local Green's formula on the $(n-k-1)$ cylinder $C_{n-k-1}$. Unfortunately, a further coupling of antipodal points does not lead to a further degree of convergence. Thus, we do not get a similar type of Green's formula for harmonic functions for the $n$-torus $T_{n}$, or for $k$-monogenic functions on the cylinders $C_{n-l}$, respectively, when $l \leq k$.

\section{References}

[1] Ahlfors, L. V.: Möbius transformations in $\mathbb{R}^{n}$ expressed through $2 \times 2$ matrices of Clifford numbers. Complex Variables Theory Appl. 5 (1986), 215-224.

[2] BeLL, S.: The Cauchy transform, potential theory and conformal mapping. Studies in Advanced Mathematics. CRC Press, Boca Raton, 1992.

[3] Bernstein, S. and Lanzani, L.: Szegö projections for Hardy spaces of monogenic functions and applications. Int. J. Math. Math. Sci. 29 (2002), no. $10,613-624$.

[4] Brackx, F., Delanghe, R. and Sommen, F.: Clifford analysis. Research Notes in Mathematics 76. Pitman, London, 1982.

[5] Calderbank, D.: Dirac operators and Clifford analysis on manifolds with boundary. Max Plank Institute for Mathematics, Bonn, preprint number 96-131, 1996.

[6] Cnops, J.: Hurwitz pairs and applications of Möbius transformations. Thesis, Ghent State University (Belgium), 1994.

[7] Cnops, J.: An Introduction to Dirac Operators on Manifolds. Progress in Mathematical Physics 24. Birkhäuser, Boston, 2002.

[8] Constales, D. and Krausshar, R. S.: Bergman kernels for rectangular domains and multiperiodic functions in Clifford analysis. Math. Methods Appl. Sci. 25 (2002), no. 16-18, 1509-1526.

[9] Eisenstein, G.: Genaue Untersuchung der unendlichen Doppelproducte, aus welchen die elliptischen Functionen als Quotienten zusammengesetzt sind, und der mit ihnen zusammenhängenden Doppelreihen (als eine neue Begründung der Theorie der elliptischen Functionen, mit besonderer Berücksichtigung ihrer Analogie zu den Kreisfunctionen). Crelle's Journal 35 (1847), 153-274.

[10] Krausshar, R. S.: Monogenic multiperiodic functions in Clifford analysis. Complex Variables Theory Appl. 46 (2001), no. 4, 337-368. 
[11] Krausshar, R. S.: Automorphic forms in Clifford analysis. Complex Var. Theory Appl. 47 (2002), no. 5, 417-440.

[12] Laville, G. and Ramadanoff, I.: Elliptic Cliffordian functions. Complex Variables Theory Appl. 45 (2001), no. 4, 297-318.

[13] Leutwiler, H.: Modified Clifford analysis. Complex Variables Theory Appl. 17 (1992), 153-171.

[14] Li, C., McIntosh, A. And Qian, T.: Clifford algebras, Fourier transforms and singular convolution operators on Lipschitz surfaces. Rev. Mat. Iberoamericana 10 (1994), 665-721.

[15] Li, C., McIntosh, A. And Semmes, S.: Convolution singular integrals on Lipschitz surfaces. J. Amer. Math. Soc. 5 (1992), 455-481.

[16] LiU, H. And RYAN, J.: Clifford analysis techniques for spherical PDE. J. Fourier Anal. Appl. 8 (2002), no. 6, 535-563.

[17] McIntosh, A.: Clifford algebras, Fourier theory, singular integrals, and harmonic functions on Lipschitz domains. In Clifford algebras in analysis and related topics (Fayetteville, AR, 1993), 33-87. Stud. Adv. Math. CRC Press, Boca Raton, FL, 1996.

[18] Mitrea, M.: Generalized Dirac operators on nonsmooth manifolds and Maxwell's equations. J. Fourier Anal. Appl. 7 (2001), 207-256.

[19] QIAn, T.: Singular integrals with monogenic kernels on the $m$-torus and their Lipschitz perturbations. In Clifford algebras in analysis and related topics (Fayetteville, AR, 1993), 157-171. Stud. Adv. Math. CRC Press, Boca Raton, FL, 1996.

[20] QIAN, T.: Fourier analysis on starlike Lipschitz surfaces. J. Funct. Anal. 183 (2001), 370-412.

[21] Ryan, J.: Iterated Dirac operators in $\mathbb{C}^{n}$. Z. Anal. Anwendungen 9 (1990), $385-401$.

[22] Ryan, J.: Conformally covariant operators in Clifford analysis. Z. Anal. Anwendungen 14 (1995), 677-704.

[23] Schoen, R. And Yau, S.-T.: Conformally flat manifolds, Kleinian groups and scalar curvature. Invent. Math. 92 (1988), 47-71.

[24] Stein, E. M.: Singular integrals and differentiability properties of functions. Princeton Mathematical Series, 30. Princeton University Press, Princeton, N. J., 1970.

[25] Stein, E. M. And Weiss, G.: Introduction to Fourier analysis on Euclidean spaces. Princeton Mathematical Series 32. Princeton University Press, Princeton, N. J., 1971.

[26] Van Lancker, P.: Clifford analysis on the sphere. PhD thesis. Ghent State University, Belgium, 1996. 
[27] VAn Lancker, P.: Clifford analysis on the sphere. In Clifford algebras and their application in mathematical physics (Aachen, 1996), 201-215. Fund. Theories Phys. 94. Kluwer Acad. Publ., Dordrecht, 1998.

Recibido: 28 de octubre de 2002 Revisado: 7 de septiembre de 2003

\author{
Rolf Sören Kraußhar \\ Department of Mathematical Analysis \\ Ghent University, Galglaan 2 \\ B-9000 Ghent, Belgium \\ krauss@cage . ugent. be \\ John Ryan \\ Department of Mathematics \\ University of Arkansas, Fayetteville \\ AR 72701, USA \\ jryan@uark.edu
}

The second author is grateful to David Calderbank for useful suggestions that led to the development of this paper. 